babies did not correlate significantly with their respective rates of occurrence, suggesting that even in areas of socioeconomic deprivation with high rates of low birthweight babies outcome may be independent of this. Extremely low birthweight infants form a high risk population but represent only about 4:1000 of all infants born. Though this study shows that such tiny infants do best when referred to a regional centre, the effect of referral may not be as pronounced in larger infants, and further studies are necessary to clarify the issue.

\footnotetext{
References

1 Cooke RWI. In utero transfer to specialist centres. Arch Dis Child 1983;58:483-4.

2 Hughes-Davies TH. Conservative care of the newborn baby. Arch Dis Child 1979;54:59-61.
}

Received 21 November 1986

\title{
Blood cultures in neonates with percutaneous central venous catheters
}

\section{R RAMANATHAN AND M DURAND}

Newborn Division of the Los Angeles County University of Southern California Medical Center, and Department of Pediatrics, University of Southern California School of Medicine, Los Angeles, United States of America

SUMMARY We compared the results of 74 pairs of blood cultures obtained simultaneously every two weeks from a peripheral vein and a percutaneously inserted central venous catheter in 38 newborns. Three babies $(7 \cdot 9 \%)$ had bacteraemia. In two of these the central catheter was colonised 48 hours beforehand.

In most of the reported cases of sepsis related to the use of central venous catheters the diagnosis was based on the natural history or on a positive result of a blood culture obtained either from a peripheral vein or through the catheter. We considered that by obtaining simultaneous blood cultures from a peripheral vein and the catheter, it might be possible to identify catheter colonisation before peripherally drawn blood samples yielded a positive culture.

\section{Patients and methods}

Thirty eight neonates, including 27 who weighed less than $1.25 \mathrm{~kg}$, with percutaneously inserted silastic central venous catheters were studied. Clinical data are summarised in Table 1. Catheters were inserted

Table 1 Clinical characteristics of the 38 infants studied. Values are mean (SD) [range]

\begin{tabular}{lccc}
\hline Birth weight (g) & 1228 & $(705)$ & {$[620-2930]$} \\
Gestational age (weeks) & $29 \cdot 2$ & $(4 \cdot 4)$ & {$[25-42]$} \\
Postnatal age (days) & $19 \cdot 6$ & $(15 \cdot 7)$ & {$[1-81]$} \\
Weight when catheter put in place $(\mathrm{g})$ & 1515 & $(790)$ & {$[650-3320]$} \\
\hline
\end{tabular}

using the peripheral veins of the extremities and scalp exclusively. ${ }^{1}$ The infusion included FreAmine III (American McGaw, American Hospital Supply Corporation) and $10 \%$ Intralipid (Cutter Laboratories). All infusions and adminstration sets were changed daily by the nurses under sterile conditions.

The $1 \mathrm{ml}$ blood samples were drawn simultaneously, one from a peripheral vein and the other through the central catheter, at two weekly intervals for as long as the catheters were in place. Each blood specimen was cultured on aerobic and anaerobic culture media. Aerobic culture medium consisted of $10 \mathrm{ml}$ tryptic soy agar broth and the anaerobic culture medium of $10 \mathrm{ml}$ brain heart infusion broth. Blood cultures were inspected daily for seven days.

\section{Results}

The mean (SD) duration that catheters were in place was $34.5(20.6)$ days, with a range of 14-95 days. Seventy four sets of blood cultures were obtained routinely. In addition, eight sets of blood cultures were drawn because of clinical suspicion of infection, but all yielded negative results. Results of the routine cultures are shown in Table 2 . Three of the 38 patients had positive blood cultures, an incidence of bacteraemia of $7.9 \%$. These three cases are described in more detail below. In the first two patients the catheter was colonised before a positive culture was obtained from peripheral blood.

Case 1. A patient with a birth weight of $2880 \mathrm{~g}$ was receiving assisted ventilation for the management of 
Table 2 Results of routine peripheral and central line blood cultures $(n=74)$ drawn from the 38 patients

\begin{tabular}{lccccccc}
\hline & $\begin{array}{l}\text { Catheter duration at time of blood } \\
\text { cultures }\end{array}$ \\
\cline { 2 - 8 } & 14 & 28 & 42 & 56 & 70 & 84 \\
\hline $\begin{array}{c}\text { No of paired blood } \\
\text { samples cultured }\end{array}$ & 38 & 19 & 11 & 3 & 2 & 1 \\
$\begin{array}{c}\text { No of positive blood cultures: } \\
\text { Peripheral vein } \\
\begin{array}{c}\text { Percutaneous central venous } \\
\text { catheter }\end{array}\end{array}$ & 1 & 0 & 0 & 0 & 0 & 0 \\
\hline
\end{tabular}

*Peripheral vein blood cultures became positive 48 hours later.

omphalocele, ventricular septal defect, and overriding aorta. Blood culture drawn through the catheter four weeks after the catheter had been put in position was positive for group $\mathrm{D}$ enterococcus, while the simultaneous blood culture from a peripheral vein yielded negative results. A repeat blood culture fron: the peripheral vein 48 hours later was positive for the same organism.

Case 2. An infant with a gestational age of 27 weeks and birth weight of $800 \mathrm{~g}$ was receiving assisted ventilation for respiratory distress syndrome. Blood culture drawn through the catheter at four weeks was positive for non-haemolytic streptococcus. Simultaneous blood culture from the peripheral vein yielded negative results. Repeat blood cultures performed through the catheter and a peripheral vein 48 hours later were both positive for nonhaemolytic streptococcus.

Both patients were treated successfully after elective catheter removal.

Case 3. This infant, who had a gestational age of 26 weeks and a birth weight of $670 \mathrm{~g}$, was on assisted ventilation for chronic lung disease. Blood cultures drawn simultaneously through the catheter and a peripheral vein at the end of two weeks were both positive for Staphylococcus epidermidis. As this infant was clinically stable we elected to treat him with antibiotics, without removal of the catheter. Subsequent blood cultures taken through the catheter and a peripheral vein 48 hours after treatment was begun yielded negative results. No further episodes of infection occurred in this patient.

\section{Discussion}

Sepsis remains the most important complication related to the use of central venous catheters. Infection rates of $6-20 \%$ have been reported in older patients; ${ }^{2}$ higher rates have been reported in newborn infants. ${ }^{34}$ To our knowledge, this is the first prospective evaluation of the use of simultaneous blood cultures, obtained from a peripheral vein and through a central catheter, during infection surveillance in sick neonates receiving parenteral alimentation through percutaneous central venous catheters. In our population we found a low incidence of bacteraemia $(7.9 \%)$. This compares favourably with previous reports in neonates and young infants receiving parenteral nutrition through surgically placed Broviac catheters $(26.6 \%$ and $31.6 \%$, respectively). ${ }^{34}$

Polymicrobial bacteraemia associated with the use of lipid emulsions in a neonatal intensive care unit had been reported by Jarvis and coworkers. ${ }^{5}$ Powell et al isolated Malassezia furfur from blood cultures drawn from Broviac catheters in five infants receiving fat emulsions through the central lines. ${ }^{6}$ They postulated that contamination of the catheter at the time of placement might be the most likely origin of infection. Despite the simultaneous use of FreAmine III and Intralipid, the incidence of basteraemia in our study did not differ from that reported by Loeff et al in neonates and young infants receiving parenteral nutrition, exclusive of fat emulsions, through small silastic catheters. ${ }^{3}$ Thus the addition of Intralipid did not influence the infection rate in our study.

A positive blood culture obtained through a central line, in the face of a simultaneous negative peripheral venous blood culture, suggests catheter colonisation. This group of infants will benefit from removal of the central venous catheters. If we had relied solely on the blood cultures from the peripheral veins we would have missed the catheter colonisation in two of our patients. Positive blood cultures obtained through the catheters enabled us to identify the onset of infection early and helped us in the management of these patients.

Our findings suggest that the use of percutaneously inserted small central venous catheters is associated with a low incidence of bacteraemia in sick neonates receiving parenteral alimentation and that in addition to peripheral venous blood cultures, percutaneous central venous catheters can be used to obtain blood cultures for the early detection of catheter colonisation in newborn infants.

\footnotetext{
References

I Durand M, Ramanathan R, Martinelli B, Tolentino M. Prospective evaluation of percutaneous central venous silastic catheters in newborn infants with birth weights of 510 to 3920 grams. Pediatrics 1986:78:245-50.

2 Weber TR, West KW, Grosfeld JL. Broviac central venous catheterization in infants and children. Am J Surg 1983;145: 202-4.
} 
${ }^{3}$ Loeff DS, Matlak ME, Black RE, Overall JC, Dolcourt JL. Johnson DG. Insertion of a small central venous catheter in neonates and young infants. J Pediatr Surg 1982:17:944-8.

4 Sadiq F, Davaskar S, Weber T, Keenan W. Life threatening complications of Broviac catheterization. Pediatr Res 1985: 19:361.

5 Jarvis WR. Highsmith AK, Allen JR, Haley RW. Polymicrobial bacteremia associated with lipid emulsion in a neonatal intensive care unit. Pediatr Infect Dis 1983;2:203-8.
${ }^{6}$ Powell DA, Aungst J. Snedden S, Hansen N. Brady M. Broviac catheter-related Malassezia furfur sepsis in five infants receiving intravenous fat emulsions. J Pediatr 1984:105:987-90.

Correspondence to Dr M Durand. LAC-USC Medical Center. Women's Hospital, Room L-919. 1240 N Mission Road. Los Angeles, California 90033. United States of America.

Received 23 December 1986

\section{Congenital hypertrichosis lanuginosa: neonatal shaving}

\section{J W PARTRIDGE}

Warneford Hospital, Leamington Spa, Warwickshire

SUMMARY A newborn baby presented with overgrowth of lanugo hair and a precocious tooth. She was shaved infrequently until aged 9 months, when spontaneous loss of body hair began. Pyloric stenosis at 6 weeks was surgically corrected. There was a family history of hypertrichosis and dental anomalies.

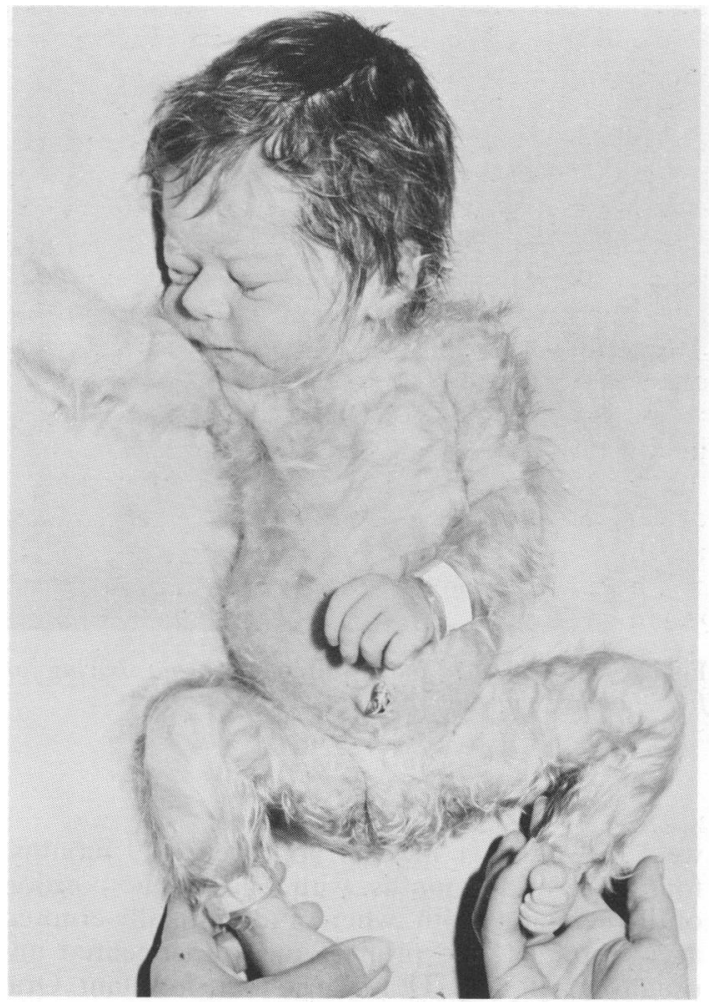

Congenital hypertrichosis lanuginosa is a rare disorder that causes persistent, excessive lanugo and dental anomalies. Neonatal and determatological textbooks do not provide guidance on immediate management: this report describes one approach, shaving.

\section{Case report}

The baby girl, the first child of unrelated parents,

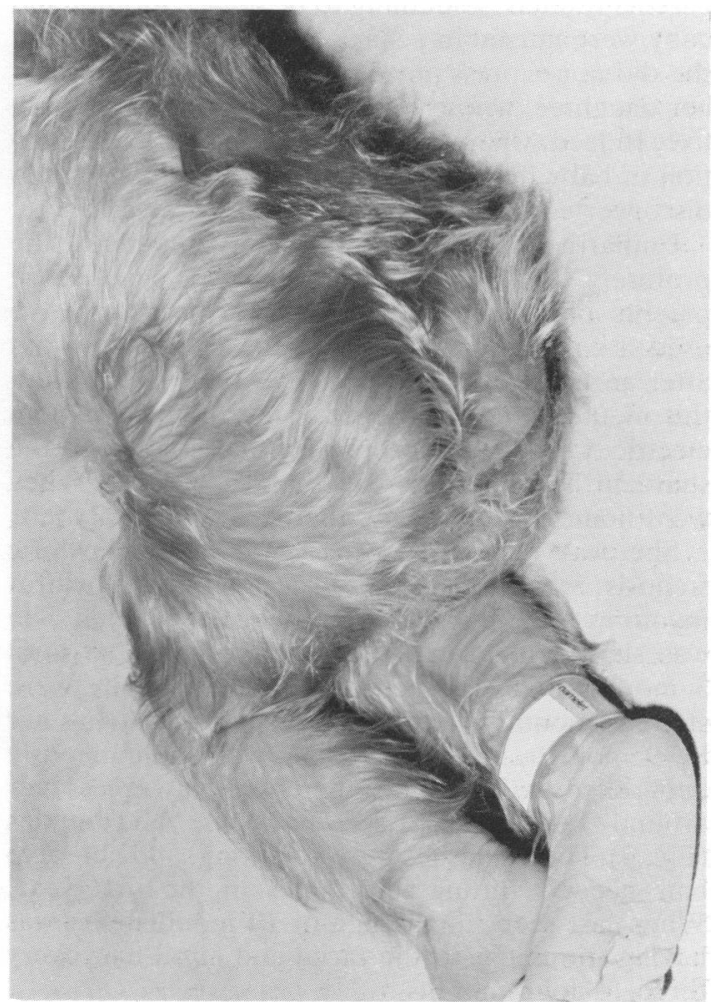

Fig. 1 A case of congenital hypertrichosis lanuginosa at presentation at 4 days of age. 